\section{Composição miofibrilar do Glúteo Médio de equinos de Concurso Completo de Equitação na fase inicial do treinamento}

\author{
Myofibrillar composition of Gluteus Medius in eventing horses \\ during the early training phase
}

\author{
Bruno Gonçalves de Souza, ${ }^{1,2 *}$, Cristiano Chaves Pessoa da Veiga', Gabriela Ferreira de Oliveira ${ }^{3}$, \\ Simone Bizerra Calado ${ }^{3}$, Juliana da Silva Leite ${ }^{4}$, Fernando Queiroz de Almeida ${ }^{5} \&$ Ana Maria Reis Ferreira ${ }^{4}$ \\ 'Médicos veterinários, DScs. Hospital Veterinário - HV, Instituto de Veterinária - IV, Universidade Federal Rural do Rio de Janeiro \\ - UFRRJ, Seropédica, RJ, Brasil \\ 2Programa de Pós-graduação em Medicina Veterinária - PPGMV, Faculdade de Veterinária - FV, Universidade Federal \\ Fluminense - UFF, Niterói, RJ, Brasil \\ ${ }^{3}$ Médicas veterinárias, DScs. Pós-graduação em Medicina Veterinária, Instituto de Veterinária - IV, Universidade Federal Rural do \\ Rio de Janeiro - UFRRJ, Seropédica, RJ, Brasil \\ ${ }^{4}$ Médica veterinária, DSc. Departamento de Clínica e Patologia - DCP, Faculdade de Veterinária - FV, Universidade Federal \\ Fluminense - UFF, Niterói, RJ, Brasil \\ ${ }^{5}$ Médico veterinário, DSc. Departamento de Medicina e Cirurgia Veterinária, Instituto de Veterinária - IV, Universidade Federal \\ Rural do Rio de Janeiro - UFRRJ, Seropédica, RJ, Brasil
}

\section{Resumo}

Determinar e conhecer a composição miofibrilar dos cavalos atletas torna possível direcioná-los melhor segundo sua aptidão física específica e certamente poupa-se tempo e recursos na preparação esportiva destes animais. Por meio da técnica de biópsia muscular percutânea e de diferentes técnicas enzimo-histoquímicas têm sido possível classificar estas fibras, utilizando estes dados na seleção destes cavalos e na condução adequada de protocolos de treinamento. Com o objetivo de avaliar a composição miofibrilar do Glúteo Médio dos cavalos selecionados para a modalidade de Concurso Completo de Equitação pela Escola de Equitação do Exército-Rj, foram utilizados treze equinos, mestiços das raças Hanoveriano, Puro Sangue Inglês e Brasileiro de Hipismo, na faixa etária de 6 a 12 anos. Todos os animais foram submetidos a procedimento de biópsia muscular percutânea e os fragmentos musculares submetidos a técnicas de enzimo-hitoquímica para distinção dos três diferentes tipos de fibras musculares por meio da avaliação da reatividade das enzimas adenosina trifosfato miosínica (mATPase) e da succinato desidrogenase (SDH). Os animais utilizados apresentaram distribuição relativa média dos diferentes tipos de fibras musculares de forma aparentemente bastante homogênea, com 25,9\%, 35,7\% e 38,4\% de fibras do tipo I, IIA e IIX, respectivamente, porém encontrando-se diferença estatística significativa, com maior proporção de fibras do tipo II em relação as do tipo I, sugerindo tratar-se de influência genética direta das raças formadoras do plantel do Exército Brasileiro.

Palavras chave: biópsia muscular, cavalo, enzimo-histoquímica.

\begin{abstract}
The studies of myofibrillar composition in athletic horses make it possible to best direct them according to their specific fitness and certainly saves time and resources in the preparation of these athletic animals. Through the technique of percutaneous muscle biopsy and different enzyme-histochemical techniques have been possible to classify these fibers in three different types, type I, IIA and IIX. This information can be applied in the selection of those horses and to the possibility the proper conduct of training protocols. In order to evaluate the myofibrillar composition of Gluteus Medius to the selected mode of Eventing by Army Riding School -RJ, thirteen horses, crossbred breeds of Hanoverian, Thoroughbred and Brazilian Equestrian, in the age range 6-12 years, were used. All animals were submitted to a percutaneous muscle biopsy procedure and the fragments subjected to enzyme-histochemical techniques for distinguishing the three different types of muscle fibers by assessing the reactivity of the enzyme adenosine triphosphate miosínica (mATPase) and succinate dehydrogenase (SDH). The animals used had an average relative distribution of different types of muscle fibers apparently quite homogeneous, with $25.9 \%$, 35.7\% and 38.4\% of fibers of type I, IIA and IIX, respectively, but lying statistically significant difference, with a higher proportion of type II fibers compared to type I, suggesting that it was a direct genetic influence of the training squad races of the Brazilian Army
\end{abstract}

Keywords: muscle biopsy, equine, enzyme-histochemistry.
Como citar: Souza, B. G., Veiga, C. C. P., Oliveira, G. F., Calado, S. B., Leite, J. S., Almeida, F. Q., \& Ferreira, A. M. R. (2017). Composição miofibrilar do Glúteo Médio de equinos de Concurso Completo de Equitação na fase inicial do treinamento. Brazilian Journal of Veterinary Medicine, 39(1), 20-27. doi: 10.29374/2527-2179.bjvm0087

Fonte de financiamento: GFO é bolsista CAPES. FQA e AMRF são bolsistas CNPq.

Conflito de interesses: Os autores declaram não haver conflito de interesses que precisam ser informados.

Recebido: Junho 23, 2016. Aceito: Novembro 15, 2016

O estudo foi realizado na Escola de Equitação do Exército - EsEqEx, Rio de Janeiro, RJ, Brasil

\section{*Correspondência}

Bruno Gonçalves de Souza Hospital Veterinário - HV, Instituto de Veterinária - IV, Universidade Federal Rural do Rio de Janeiro - UFRRJ

BR 465, Km 7, Campus Seropédica CEP 23890-000 - Seropédica (RJ), Brasil E-mail: brunomedvet@yahoo.com.br
Copyright Souza et al. Esteé um artigo publicado em acesso aberto (Open Access) sob a licença Creative Commons Attribution Non-Commercial, que permite uso, distribuição e reprodução em qualquer meio, sem restrições desde que sem fins comerciais e que o trabalho original seja corretamente citado. 


\section{Introdução}

O tecido muscular dos equinos é bastante heterogêneo, apresentando estruturas contráteis ou miofibrilares com características bioquímicas e metabólicas bastante diferentes entre si e encontradas em diferentes proporções dependendo do músculo avaliado e de sua função motora predominante (Valberg \& Éssen-Gustavsson, 1987; Serrano \& Lopez-Rivero, 2000). A composição miofibrilar dos equinos é determinada geneticamente e pode sofrer influência de diversos fatores, tais como: raça, sexo, idade, e, especialmente no caso de equinos atletas, o treinamento ao qual são submetidos (Ronëus et al., 1991).

Conhecer a composição miofibrilar do cavalo atleta torna possível avaliar as respostas individuais do animal ao treinamento ao qual é submetido e melhor direcioná-los segundo sua aptidão física específica. Desta forma certamente poupa-se tempo e recursos na preparação dos animais, que passam a ser aplicados em animais com chances reais de vitória de acordo com sua aptidão genética e a modalidade equestre escolhida (Lindner \& Boffi, 2007).

A partir da técnica de biópsia muscular percutânea, introduzida no estudo da fisiologia muscular de equinos por Lindholm \& Piehl (1974), e de diferentes técnicas laboratoriais têm sido possível classificar as fibras musculares dos cavalos (Lopez-Rivero et al., 1992). Estas fibras são primariamente classificadas quanto à expressão das isoformas da cadeia pesada da miosina (CPM) em CPM I ou CPM II, que determinam características como velocidade de contração e resistência, sendo possível distinguir duas subpopulações de fibras, uma de contração lenta (tipo I) e outra de contração rápida (tipo II) (Lopez-Rivero et al., 1996a, 1996b).

As fibras do tipo I expressam a isoforma I da CPM e apesar de possuírem baixa velocidade de contração, são capazes de contraírem repetidamente, com força moderada, por longos períodos. Estas fibras utilizam preferencialmente vias metabólicas oxidativas, consumindo basicamente ácidos graxos pra geração de energia, sendo muito resistentes à fadiga. De outro modo, as fibras do tipo II, que expressam a isoforma II da CPM, contraem mais rapidamente e são capazes de desenvolver muito mais força. Contudo, possuem menor resistência à fadiga e são mais ricas em glicogênio, evidenciando que nestes tipos de fibras, há predominância da via glicolítica do metabolismo energético (Flück \& Hoppeler, 2003; Peixoto, 2004).

As diferenças na expressão das isoformas da CPM nas fibras musculares de cavalos contribuem significativamente para as diferenças na contractilidade e propriedades metabólicas entre os tipos de fibras musculares desses animais, com reflexos diretos em sua aptidão física (Lopez-Rivero et al., 1996b). As características metabólicas destas fibras possuem estreita relação com seu tipo primário e podem ser determinadas mediante análises enzimo-histoquímicas que indiquem a capacidade oxidativa e/ou glicolítica das fibras (Éssen-Gustavsson, 1991).

A técnica enzimo-histoquímica mais utilizada na classificação dos diferentes tipos de fibras musculares de equinos é realizada com base na avaliação da atividade da ATPase miofibrilar nas fibras musculares destes animais, incubadas em diferentes pHs (Essén-Gustavsson et al., 1989), porém outras técnicas enzimáticas têm importante papel na histoquímica para esta mesma finalidade, dentre elas a técnica de determinação da reatividade da enzima succinato desidrogenase (SDH). Esta enzima está presente na mitocôndria e no retículo endoplasmático e sua reatividade reflete a capacidade de oxidação intracelular. A SDH é frequentemente utilizada como um marcador enzimático de mitocôndria e devido às próprias características metabólicas as fibras do tipo I coram-se mais intensamente com esta técnica, seguidas pelas fibras do tipo IIA com coloração intermediária e pelas do tipo IIX, que devido ao predomínio da via glicolítica possuem menor densidade mitocondrial (Dubowitz et al., 1985).

Gautheir (1969) citado por Klont et al. (1998), determinou a capacidade oxidativa da fibra muscular utilizando como marcador a enzima succinato desidrogenase (SDH) e identificou três tipos de fibras, as quais denominou segundo a tonalidade predominante, como fibras vermelhas, intermediárias e brancas. Ashmore \& Doerr (1971) associaram técnicas histoquímicas de reatividade da mATPase e da enzima SDH e descreveram três tipos de fibras: $\mathrm{mATPase} \mathrm{resistente} \mathrm{e} \mathrm{metabolismo}$ oxidativo (tipo I), mATPase ácido lábil e metabolismo oxidativo-glicolítico (tipo IIA) e mATPase ácido lábil e metabolismo glicolítico (tipo IIX).

Este estudo foi conduzido com o objetivo de determinar a composição miofibrilar do músculo Glúteo Médio de cavalos praticantes da ainda pouco estudada modalidade equestre de Concurso Completo de Equitação. 


\section{Material e métodos}

Este estudo foi conduzido no Laboratório de Avaliação do Desempenho de Equinos - LADEQ localizado na Escola de Equitação do Exército (EsEqEx), na Vila Militar, em Deodoro, Rio de Janeiro.

Foram utilizados treze equinos, mestiços das raças Hanoveriano, Puro Sangue Inglês e Brasileiro de Hipismo, na faixa etária de 6 a 12 anos, sendo nove machos castrados e quatro fêmeas, todos utilizados na disciplina de Concurso Completo de Equitação da EsEqEx.

Todos os animais foram submetidos a procedimento de biópsia muscular percutânea. As biópsias foram realizadas no antímero esquerdo dos animais, na interseção entre o terço cranial e o terço médio de uma linha imaginária ligando a tuberosidade coxal e a inserção da cauda, sobre a garupa dos equinos.

Após contenção física do animal em tronco específico, tricotomia e antissepsia do local escolhido, foi realizada anestesia local infiltrando-se, em forma de botão anestésico, $3 \mathrm{~mL}$ de solução de lidocaína a $2 \%$ sem vasoconstritor. Após aguardar aproximadamente cinco minutos e realizar nova antissepsia, foi realizada uma incisão de aproximadamente $1 \mathrm{~cm}$ de comprimento na pele e tecido subcutâneo até a fáscia glútea, utilizando-se para tal bisturi e lâmina n. ${ }^{\circ} 24$, estéreis.

Com o auxílio de uma agulha de biópsia do tipo Bergstrom 6,0 mm, foram biopsiados, a $6 \mathrm{~cm}$ de profundidade, dois fragmentos do músculo. A biópsia muscular percutânea por agulha especial foi realizada segundo metodologia descrita por Lindholm \& Piehl (1974).

Os fragmentos biopsiados foram pré-secados, rolando-os levemente sobre papel filtro com o intuito de diminuir a umidade e minimizar os efeitos deletérios do congelamento nas amostras. Em seguida, foram acondicionados em envelopes confeccionados com folhas de papel alumínio previamente identificados e imediatamente congelados em nitrogênio líquido a - $160^{\circ} \mathrm{C}$ para posterior análise enzimo-histoquímica (Figura 1).

As amostras foram submetidas a técnicas de enzimo-hitoquímica para distinção dos três diferentes tipos de fibras musculares por meio da avaliação da reatividade de determinadas enzimas. Foram realizadas as técnicas da adenosina trifosfato miosínica (mATPase) e da succinato desidrogenase $(\mathrm{SDH})$.

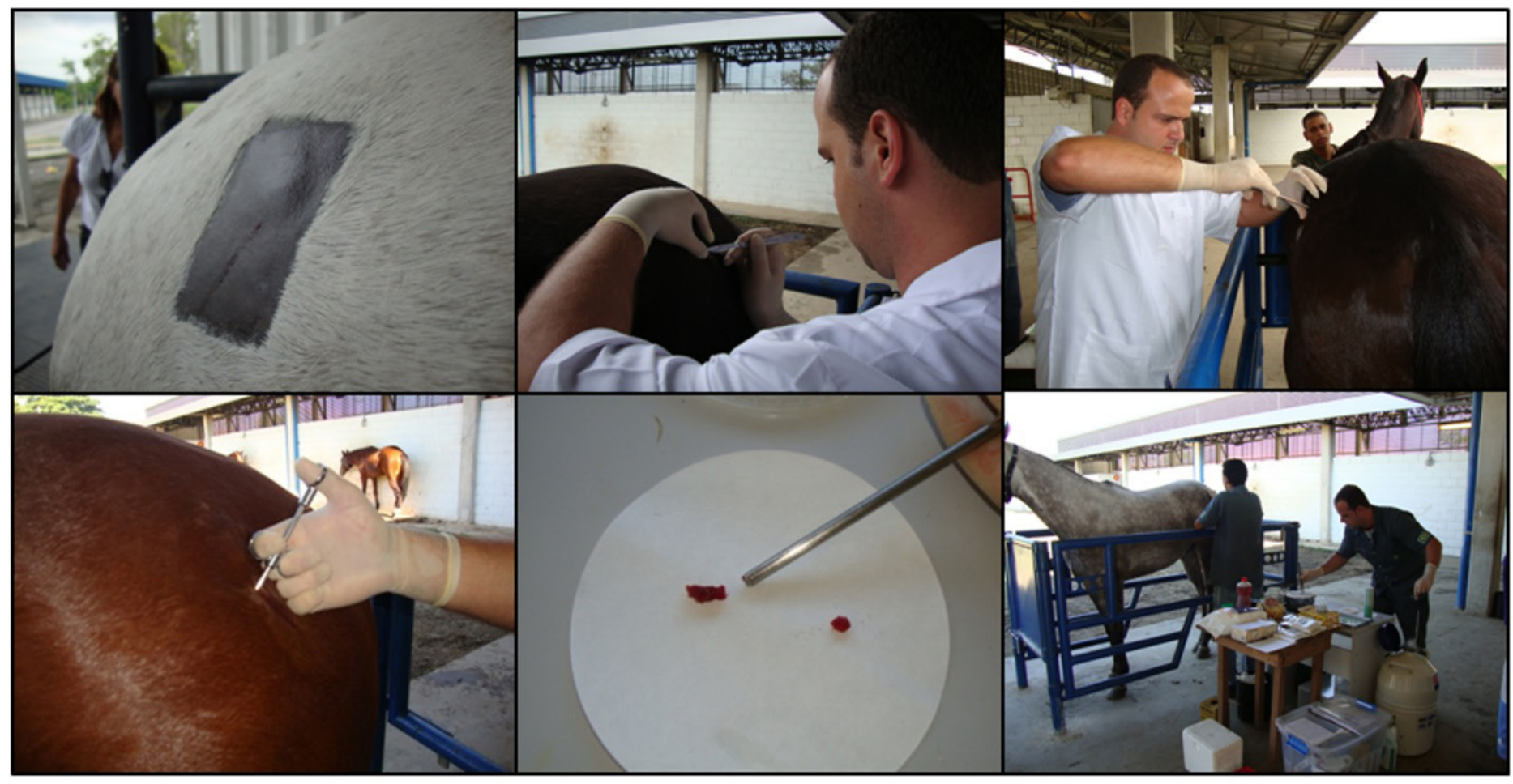

Figura 1. Sequência dos procedimentos da biópsia muscular percutânea do Glúteo Médio dos equinos: bloqueio local; incisão da pele e tecido subcutâneo; introdução da agulha de biópsia; abertura da janela de corte a 6 cm de profundidade; pré-secagem do material obtido, utilizando-se o papel filtro; identificação e congelamento das amostras. 
A técnica utilizada para essa análise foi uma adaptação do método de coloração metacromática da atividade da ATPase em miofibras, descrito por Ogilvie et al. (1990) e modificada por Ferreira(2000).

A técnica enzimo-histoquímica da mATPase, especialmente quando utilizada à incubação em $\mathrm{pH}$ 4.6, possibilitou a diferenciação dos três diferentes tipos de fibras musculares dos cavalos (Figura 2). Nos casos onde a distinção das tonalidades de castanho não era tão evidente com esta técnica, realizou-se a diferenciação das fibras, especialmente as do tipo II em IIA e IIX, utilizando-se a comparação de campos da técnica da mATPase com os cortes seriados submetidos a técnica da SDH (Figura 3), e assim foi possível determinar as proporções relativas de cada tipo de fibra muscular em todos os animais deste estudo.

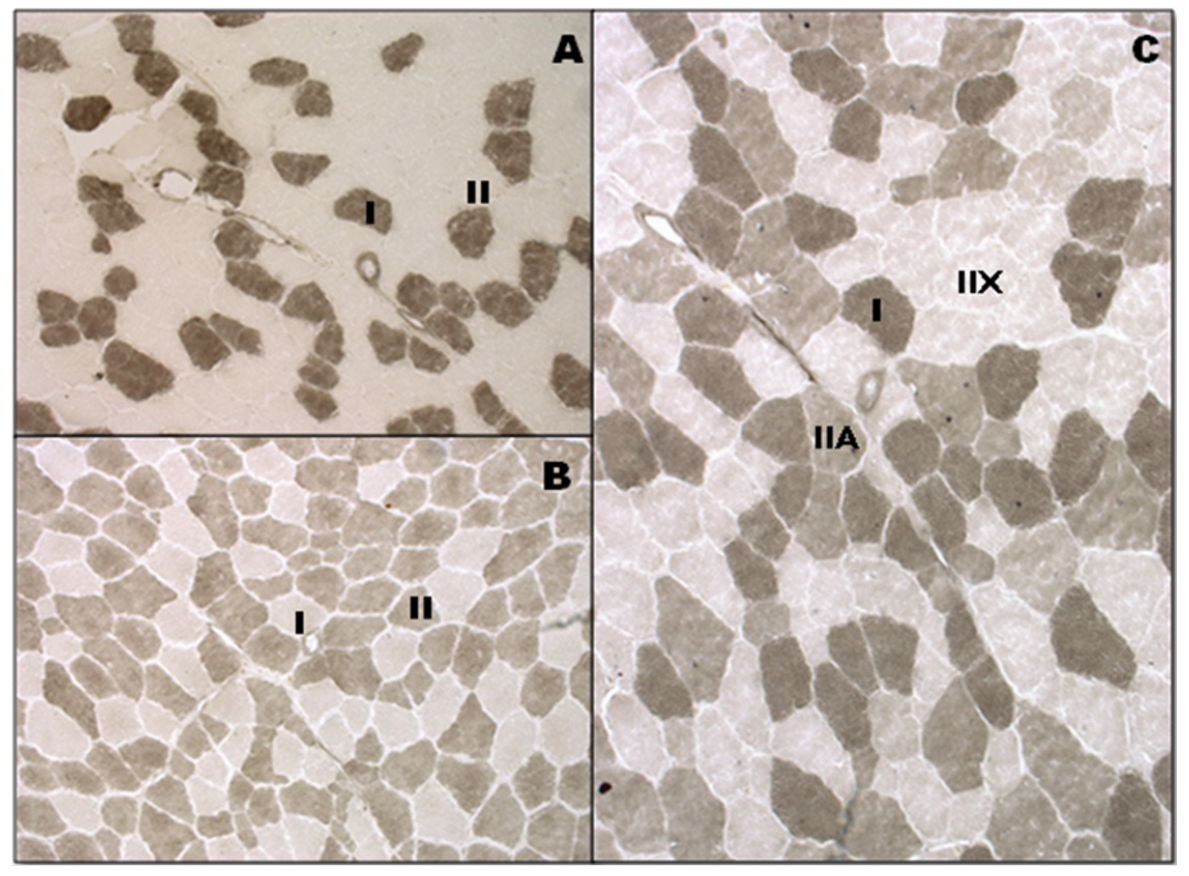

Figura 2. Fotomicrografia de cortes transversais seriados do músculo Glúteo Médio, utilizando-se a técnica de enzimo-histoquímica paramarcação daATPasemiosínica (mATPase) comincubações em diferentes pH's. (A)incubação ácida (pH 4.3), fibras do tipo I escuras e do tipo II claras; (B) incubação alcalina (pH 9.4), fibras do tipo I claras e do tipo II escuras; (C) incubaçãoácida (pH4.6), fibras do tipo I escuras, fibras do tipo IIA com tonalidadeintermediáriae fibras do tipo IIX claras. Observar o padrão em mosaico das fibras. Animal: O7. Objetivas 20X (A, B); 40X (C).

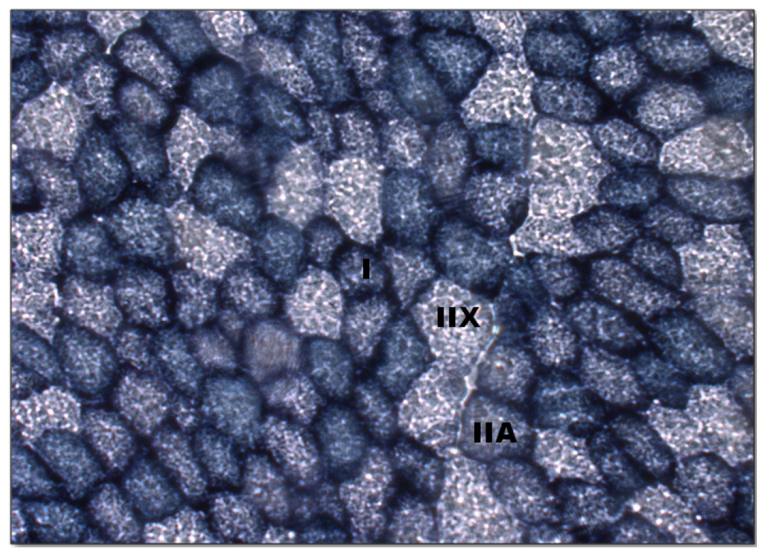

Figura 3. Fotomicrografia de corte transversal do músculo Glúteo Médio do equino de número 5, utilizando-se a técnica de enzimo-histoquímica para marcação da atividade da enzima succinato desidrogenase, onde as fibras do tipo I são coradas de azul escuro, as do tipo IIA são coradas de azul intermediário e as fibras do tipo IIX coram-se de azul claro. Obj. 20X. 
Inicialmente realizou-se a classificação e a contagem de cada tipo de fibra muscular utilizando-se as lâminas submetidas à técnica da mATPase após incubação em $\mathrm{pH}$ 4,3 ou 9,4, onde foi possível diferenciar primariamente as fibras em tipos I e II. Utilizando-se as lâminas submetidas à técnica da mATPase após incubação em pH 4,6 e, quando necessário, também recorrendo a comparação de campos seriados submetidos à técnica da SDH, realizou-se a diferenciação e contagem das fibras do tipo II em IIA e IIX.

Os resultados obtidos foram primariamente submetidos a procedimentos de estatística descritiva, com a determinação das respectivas médias e desvios-padrão e em seguida submetidos à análise de variância, sendo as médias comparadas pelo teste t de Student a 5\% de significância.

\section{Resultados}

Os cavalos de Concurso Completo de Equitação utilizados neste estudo apresentaram distribuição relativa média dos diferentes tipos de fibras musculares de forma aparentemente bastante homogênea, com 25,9, 35,7 e 38,4\% de fibras do tipo I, IIA e IIX, respectivamente, porém encontrando-se diferença estatística significativa, com maior proporção de fibras do tipo II em relação as do tipo I.

Os resultados desta distribuição em relação aos diferentes tipos de fibras musculares estão discriminados de forma individualizada na Tabela 1.

\section{Discussão}

Como relatado por diversos pesquisadores (Essén-Gustavsson et al., 1984; Lopez-Rivero et al., 1995a, 1995b; Lindner et al., 2002), o método de biópsia muscular por agulha percutânea tipo Bergström adotado neste estudo demonstrou ser uma técnica de fácil aplicação, causando lesões de pequena proporção, cuja cicatrização evoluiu de forma rápida sem a ocorrência de complicações, podendo ser aplicada inclusive em animais que estejam participando de programas de treinamento.

Conforme descrito em estudos prévios na espécie equina, os resultados obtidos a partir da técnica de mATPase permitiu confirmar a existência de três tipos de fibras puras no músculo

Tabela 1. Frequência fenotípica relativa dos três diferentes tipos de fibras musculares dos cavalos de Concurso Completo de Equitação.

\begin{tabular}{cccc}
\hline \multirow{2}{*}{ Animal: } & \multicolumn{3}{c}{ Frequência dos tipos de fibras tipo (\%) } \\
\cline { 2 - 4 } & I & IIA & IIX \\
\hline 01 & 14,1 & 45,2 & 40,7 \\
02 & 30,5 & 32,1 & 37,4 \\
03 & 26,8 & 29,7 & 43,5 \\
04 & 27,7 & 39,2 & 33,1 \\
05 & 25,1 & 32,6 & 41,5 \\
06 & 29,7 & 35,9 & 34,4 \\
07 & 33,7 & 36,5 & 29,8 \\
08 & 22,2 & 38,1 & 39,7 \\
09 & 24,3 & 37,0 & 38,7 \\
10 & 24,1 & 36,1 & 39,8 \\
11 & 26,2 & 34,7 & 39,1 \\
12 & 30,7 & 27,0 & 42,3 \\
13 & 22,2 & 38,6 & 39,2 \\
Média: & $25,9^{\mathrm{a}}$ & $35,7^{\mathrm{b}}$ & $38,4^{\mathrm{b}}$ \\
C.V. (\%): & 19,23 & 12,91 & 10,09 \\
\hline
\end{tabular}

Letras diferentes diferem significativamente pelo teste t de student $(p \leq 0,05)$. C.V. = Coeficiente de Variação. 
Glúteo Médio dos animais, as fibras tipo I, IIA e IIX. Entretanto, em alguns cortes histológicos submetidos a esta técnica, especialmente nos que foram incubados em $\mathrm{pH}$ intermediário de 4,6, não foi possível fazer a diferenciação entre as fibras do tipo II em IIA e IIX, sendo então realizada a comparação entre campos seriados de cortes submetidos a esta técnica com os submetidos a da SDH (Dubowitz et al., 1985; Serrano \& Lopez-Rivero, 2000).

Mediante a utilização da técnica enzimo-histoquímica da SDH observou-se que as fibras tipo I e IIA reagiram mais intensamente, demonstrando, portanto, serem oxidativas, enquanto as fibras do tipo IIX reagiram fracamente por apresentarem metabolismo predominantemente glicolítico. A associação das técnicas mATPase e SDH, possibilitou a diferenciação e a determinação das proporções relativas de cada um dos três tipos de fibras musculares, especialmente nos casos onde as lâminas da mATPase a 4.6 não proporcionaram uma adequada diferenciação (Dubowitz et al., 1985).

Os cavalos utilizados para a modalidade de Concurso Completo de Equitação pela EsEqEx, apresentaram uma distribuição relativamente homogênea dos três diferentes tipos de fibras musculares, com 25,9\%, 35,7\% e 38,4\% de fibras do tipo I, IIA e IIX, respectivamente. Apesar disso, detectou-se diferença significativa nesta comparação, demonstrando haver uma predominância das fibras do tipo II em relação às do tipo I. Estes resultados são bastante semelhantes aos descritos por Islas et al. (1998), que realizou biópsias musculares do músculo Glúteo Médio de 10 cavalos, mestiços para salto, meio sangue PSI, meio sangue Hackney, todos com 4 anos de idade, descrevendo proporções relativas de 26,9\%, 36,1\% e 37\% de fibras do tipo I, IIA e IIX, respectivamente.

D`Angelis et al. (2006) ao realizar estudo semelhante, utilizaram 21 equinos, entre machos e fêmeas, da raça Brasileiro de Hipismo. Seus resultados apontaram que as fibras do tipo IIX ocorrem com maior frequência, seguidas das fibras tipo IIA e, por último, as do tipo I no músculo Glúteo Médio de cavalos dessa raça, o que segundo os autores, parece ser decorrente da grande influência genética da raça PSI na formação do cavalo Brasileiro de Hipismo.

Rietbroek et al. (2006) utilizou 16 potros Hanoverianos, selecionados para a modalidade de adestramento e descreveram as seguintes proporções de fibras musculares: $24.4 \%$ de fibras do tipo I, 42.6\% de fibras do tipo IIA e 29.4\% de fibras do tipo IIX. Estes autores ressaltaram ainda, a importância da predominância das fibras do tipo IIA para os cavalos que disputam esta modalidade equestre, especialmente em copetições de alto nível, como é o caso dos animais da raça Andaluz, que apresentam maiores proporções de fibras do tipo IIA, que além de apresentarem contração rápida, possuem uma considerável resistência à fadiga (Lopez-Rivero et al., 1990, 1995a).

Como o plantel da EsEqEx conta apenas com animais provenientes do cruzamento das raças PSI, Hanoveriano e BH, em diferentes graus de sangue, acredita-se que as proporções relativas dos três diferentes tipos de fibras musculares encontradas nestes animais, tenha sofrido grande influência genética, evidenciada pela predominância de fibras do tipo IIA e IIX, como frequentemente descrito na literatura para animais das raças supracitadas (Rietbroek et al., 2006; D’Angelis et al., 2006; Islas et al., 1998). Além disso, considera-se que esta homogeneidade na distribuição dos diferentes tipos de fibras musculares de cavalos de Concurso Completo de Equitação (CCE) seja uma característica desejável já que esta modalidade não premia o cavalo apenas por sua velocidade ou resistência exuberantes, e sim o mais completo, aquele que consegue combinar qualidades que o torne, não apenas excelente em umas das provas, mas sim, equilibrado e bom nos três tipos distintos de provas realizadas no CCE (Marlin, 2001).

\section{Conclusão}

Apesar da distribuição aparentemente homogênea quanto aos tipos de fibras musculares, os cavalos de CCE da EsEqEx, durante a fase inicial do período de treinamento, apresentaram proporções relativas de fibras musculares do tipo II superiores as do tipo I, indicando tratar-se de influência genética direta das raças formadoras do plantel do Exército Brasileiro.

\section{Referências}

Ashmore, C. R., \& Doerr, L. (1971). Comparative aspects of muscle fiber types in different species. Experimental Neurology, 31(3), 408-418. http://dx.doi.org/10.1016/0014-4886(71)90243-3. PMid:4254914. 
Dubowitz, V., Sewry, C. A., \& Fitzsimmons, R. B. (1985). Muscle biopsies: a practical approach (2nd ed., 720 p.). Philadelphia: Baillere Tindall.

Essén-Gustavsson, B. (1991). Training effect on skeletal muscle. In P. Kallings. Proceedings of the International Conference on Equine Sports Medicine (pp. 18-23). Cambridge, United Kingdom.

Essén-Gustavsson, B., Karlström, K., \& Lindholm, A. (1984). Fibre types, enzyme activities and substrate utilization in skeletal muscles of horses competing in endurance rides. Equine Veterinary Journal,16(3), 197-202. http:// dx.doi.org/10.1111/j.2042-3306.1984.tb01903.x. PMid:6734585.

Essén-Gustavsson, B., McMiken, D., Karlström, K., Lindholm, A., Persson, S., \& Thornton, J. (1989). Muscular adaptation of horses during intensive training and detraining. Equine Veterinary Journal, 21(1), 27-33. http:// dx.doi.org/10.1111/j.2042-3306.1989.tb02085.x. PMid:2920697.

Ferreira, A. M. R. (2000). Análise histoquímica e imuno-bistoquímica de macromoléculas da matriz extracelulare dos tipos de fibras do músculo glúteo médio equino. Universidade Federal Fluminense. Faculdade de Medicina, Niterói.

Flück, M., \& Hoppeler, H. (2003). Molecular basis of skeletal muscle plasticity-from gene to form and function. Reviews of Physiology, Biochemistry and Pharmacology, 146, 159-216. PMid:12605307.

Islas, A., Quezada, M., Bernales, A., Mora, G., Lopez-Rivero, J. L., Merino, V., Briones, M., Escalona, C., \& Marin, L. (1998). Características histoquímicas y morfométricas del Gluteus Medius em eqüinos em preparación para competências de salto. Estudo preliminar. Archivos de Medicina Veterinaria, 30(1). http://dx.doi.org/10.4067/ S0301-732X1998000100011.

Klont, R. E., Brocks, L., \& Eikelenboom, G. (1998). Muscle fiber type and meat quality. Meat Science, 49, $219-229$. http://dx.doi.org/10.1016/S0309-1740(98)90050-X. PMid:22060713.

Lindholm, A., \& Piehl, K. (1974). Fibre composition, enzyme activity and concentrations of metabolites and electrolytes in muscles of Standardbred horses. Acta Veterinaria Scandinavica, 15(3), 287-309. PMid:4137664.

Lindner, A., \& Boffi, F. M. (2007). Pruebas de ejercicio. In F. M. Boffi. Fisiologia Del Ejercicio en Equinos (pp. 243254, cap. 17). Inter-medica, Buenos Aires, Argentina.

Lindner, A., Dag, S., Marti-Korff, S., Quiroz-Rothe, E., López Rivero, J. L., \& Drommer, W. (2002). Effects of repeated biopsying on muscle tissue in horses. Equine Veterinary Journal, 34(6), 619-624. http://dx.doi.org/10.2746/0 42516402776180188. PMid:12358004.

Lopez-Rivero, J. L., Agüera, E., Monterde, J. G., Diz, A., \& Vivo, J. (1990). Fibre size and composition in the middle gluteal muscle of the Andalusian horse. Equine Veterinary Journal, 22(4), 286-287. http://dx.doi. org/10.1111/j.2042-3306.1990.tb04269.x. PMid:2209526.

Lopez-Rivero, J. L., Agüera, E., Monterde, J. G., Vivo, J., \& Rodríguez-Barbudo, M. V. (1992). Skeletal muscle fiber size in untrained and endurance-trained horse. American Journal of Veterinary Research, 53(5), 847-850. PMid:1524314.

Lopez-Rivero, J. L., Serrano, A. L., \& Henckel, P. (1995a). Activities of selected aerobic and anaerobic enzymes in the gluteus medius of endurance horses with different performance records. Veterinary Research, 137(8), 187-192. PMid:8560724.

Lopez-Rivero, J. L., Ruz, M. C., Serrano, A. L., \& Diz, A. M. (1995b). Effects of the 3-month endurance training programme on skeletal muscle histochemistry in Andalusian, Arabian and Anglo-Arabian horses. Equine Veterinary Journal, 27(1), 51-59. http://dx.doi.org/10.1111/j.2042-3306.1995.tb03033.x. PMid:7774548.

Lopez-Rivero, J. L., Talmadge, R. J., \& Edgerton, V. R. (1996a). Myosin heavy chain isoforms in adult equine skeletal muscle: An immunohistochemical and electrophoretic study. The Anatomical Record, 246(2), 185-194. http:// dx.doi.org/10.1002/(SICl)1097-0185(199610)246:2<185::AID-AR5>3.0.CO;2-0. PMid:8888960.

Lopez-Rivero, J. L., Talmadge, R. J., \& Edgerton, V. R. (1996b). Correlation between myofibrillar ATPase activity and myosin heavy chain composition in equine skeletal muscle and the Influence of training. The Anatomical Record, 246(2), 195-207. http://dx.doi.org/10.1002/(SICI)1097-0185(199610)246:2<195::AID-AR6>3.0.CO;2-0. PMid:8888961.

Marlin, D. J., Schroter, R. C., Mills, P. C., White, S. L., Maykuth, P. L., Votion, D., \& Waran, N. (2001). Performance of acclimatized European Horses in modified one star three-day event in heat and humidity. Journal of Equine Veterinary Science, 21(7), 341-350. https://doi.org/10.1016/S0737-0806(01)70077-9.

Ogilvie, R. W., \& Feeback, D. L. Olgivie, R. W., \& Feeback, D. L. (1990). A metachromatic dye-ATPase method for the simultaneous identification of skeletal muscle fiber types I, IIA, IIB, IIC. Stain Technology, 65(5), 231-241. http://dx.doi.org/10.3109/10520299009105613. PMid:1703671.

Peixoto, F. J. G. (2004). Adaptações bioquímicas em fibras musculares esqueléticas de eqüinos treinados para enduro: correlação entre tipagem muscular e expressão da isoforma neuronal da óxido nítrico sintase (170 p.). Campinas: Universidade Estadual de Campinas.

Rietbroek, N. J., Dingboom, E. G., \& Everts, M. E. (2006). Developmental changes and the effect of selection on the equine locomotory muscle. In $7^{\text {th }}$ Proceedings of the Muscle Physiology and Energetics Session (pp. 79). ICEEP, Fontainebleau, França.

Ronëus, M., Lindholm, A., \& Åsheim, Å. (1991). Muscle characteristics in Thoroughbreds of different ages and sexes. Equine Veterinary Journal,23(3), 207-210. http://dx.doi.org/10.1111/j.2042-3306.1991.tb02757.x. PMid:1884703. 
Serrano, A. L., \& Lopez-Rivero, J. L. (2000). Myosin heavy chain profile of equine gluteus medius muscle following draught-exercise training and detraining. Journal of Muscle Research and Cell Motility, 21(3), 235-245. http:/l dx.doi.org/10.1023/A:1005642632711. PMid:10952171.

Valberg, S., \& Éssen-Gustavsson, B. (1987). Metabolic response to racing determined in pools of type I, IIA and IIB fibers. Equine Exercise Physiology, 2, 290-301. 